Int. J. Odontostomat.,

11(1):77-82, 2017.

\title{
Tracción de Canino Maxilar Izquierdo Impactado con Botón Bondeable, Ligadura Metálica y Cadena Elastomérica
}

\author{
Traction of Left Maxillary Impacted Canine with Bondeable Button, \\ Metal Ligature and Elastomeric Chain
}

\author{
Erika Orozco Estrada1; Beatriz Gurrola Martínez ${ }^{2}$ \& Adán Casasa Araujo ${ }^{3}$
}

OROZCO, E. E.; GURROLA M. B. \& CASASA, A. A. Tracción de canino maxilar izquierdo impactado con botón bondeable, ligadura metálica y cadena elastomérica. Int. J. Odontostomat., 11(1):77-82, 2017.

RESUMEN: Se presenta una paciente con 13 años y 7 meses de edad, el motivo de consulta fue "un colmillo en el paladar"; es clase I molar bilateral, clase I canina derecha con el canino infantil 53, clase II canina derecha, clase I canina izquierda, clase I esquelética, normodivergente, tiene apiñamiento dental moderado superior e inferior leve, mordida cruzada anterior de canino superior derecho, diente 23 impactado, acortamiento radicular en los incisivos centrales y segundos premolares inferiores. El tratamiento consistió en la extracción de dientes temporales 53 y 63 , alineación nivelación, tracción quirúrgica del diente 23 , cierre de espacios, detallado, retención. La retención se llevó a cabo mediante retenedores "Hawley" superior con "Fingers" en los dientes 23 y 13 e inferior. El tiempo de tratamiento activo fue 1 años 2 meses.

PALABRAS CLAVE: canino impactado, tracción quirúrgica.

\section{INTRODUCCION}

La formación del canino superior comienza a los cuatro o cinco meses de edad y el esmalte se forma en su totalidad entre los seis y siete años, erupciona en promedio a los 11,6 años y su raíz queda formada totalmente a los 13,6 años de edad. El canino inferior tiene una formación muy semejante, su erupción se realiza a los 10,6 años de edad y su raíz queda formada completamente a los 12 años (Esquivel, 1978; Lucea, 2005). Una retención dental es aquel estado en el cual un diente, parcial o totalmente desarrollado, queda alojado en el interior de los maxilares después de haber pasado la época promedio normal de su erupción. La etiología es multifactorial, dentro de las cuales podemos mencionar: discrepancia de tamaño dental y de longitud del arco, retención prolongada o pérdida prematura del canino deciduo, posición anormal del germen dentario, anquilosis, problemas nasorespiratorios, presencia de una hendidura alveolar, patologías localizadas como quistes, neoplasias, odontomas, etc. (Proffit et al., 2008). Se denominan dientes retenidos cuando se encuentran dentro del hueso con su saco epitelial íntegro, sin comunicación con la cavidad bucal y sin que llegue su momento de erupción (Ugalde, 2001). En ortodoncia con mucha frecuencia, se enfrentan problemas oclusales con la retención de los caninos maxilares y en algunas ocasiones los mandibulares, por lo que es importante tener conocimientos claros acerca de la etiología, el diagnóstico y el tratamiento. Algunos conceptos de caninos retenidos: Caninos retenidos, no hacen erupción debido a obstáculos mecánicos o a falta de fuerza eruptiva (Uribe, 2009). Diente que no ha perforado la mucosa bucal y por lo tanto no ha adquirido una posición normal en el maxilar (Ballesteros, 2016). El tiempo de intervención en el paciente con caninos retenidos es crítico, razón por la cual es necesario una eva-

\footnotetext{
${ }^{1}$ Egresada de la maestría de Ortodoncia y Ortopedia maxilofacial del Centro de Estudios Superiores en Ortodoncia (CESO), México.

2 Profesor del CESO y profesor de tiempo completo Titular "C" en la carrera de Cirujano Dentista de la Facultad de Estudios Superiores Zaragoza UNAM, México.

${ }^{3}$ Director del CESO, México.
} 
luación cuidadosa del estado de desarrollo de la dentición (Gomez et al., 2002). El pronóstico del movimiento ortodóncico de un diente retenido depende de una variedad de factores, tales como la posición del diente retenido con respecto a los dientes vecinos, su angulación, la distancia que el diente debe recorrer y la posible presencia de anquilosis. En general, los caninos retenidos horizontalmente o anquilosados son los más difíciles de manejar y tienen el peor pronóstico (Gange, 2016).

\section{REPORTE DE CASO}

En las fotografías extraorales en la de frente sonrisa tenemos simetría facial y la línea media dental superior coincidente con la línea media facial (Fig. 1A) y en la extraoral lateral el perfil recto (Fig. 1B).

Evaluación inicial la fotografía intraoral de frente se ven las líneas dental inferior desviada a la derecha con respecto a la línea media dentaria superior, el apiñamiento moderado superior y leve inferior (Fig. 2A) en el lado derecho, se observa una relación molar de clase I y la relación canina clase I con el canino OD 53 y clase II con el OD 13 (Fig. 2B). En la fotografía intraoral de lado izquierdo, vemos una relación molar de clase I y relación canina de clase I (Fig. 2C).

Técnica y mecánica: Se tomaron los estudios radiográficos de inicio: los cuales comprendieron, la radiografía lateral de cráneo donde se ve la clase I esquelética de la paciente, y que es un paciente normodivergente (Fig. 3A). En la radiografía panorámica tenemos dentición mixta por la presencia de los caninos infantiles 53 y 63, así como los 32 dientes permanentes, y la posición del diente 23 esta impactado, los terceros molares están en formación (Fig. 3B). En las radiografías periapicales donde se puede observar el acortamiento radicular de los dientes 11 y 21 (Fig. 3C), en Fig. 3D, la raíz corta de los primeros premolares.

En este caso se realizó una segunda fase, que consistió en un procedimiento quirúrgico. A continuación los pasos que se siguieron para descubrir el canino que estaba en el paladar, vemos el colgajo palatino y el canino impactado (Fig. 4A) y la técnica a seguir; en Fig. 4B, el levantamiento del colgajo y el canino en posición, en Fig. 4C, la tracción quirúrgica con el alambre y colocación del botón en el canino OD 23, y finalmente en Fig. 4D, los puntos de sutura en el paladar.

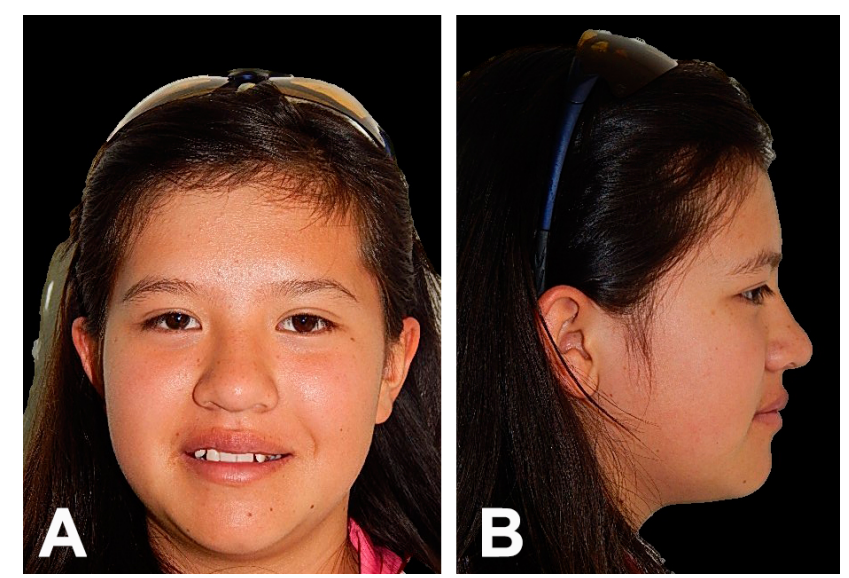

Fig. 1.A. Vista extroral frontal; 1. B. Vista extraoral perfil.
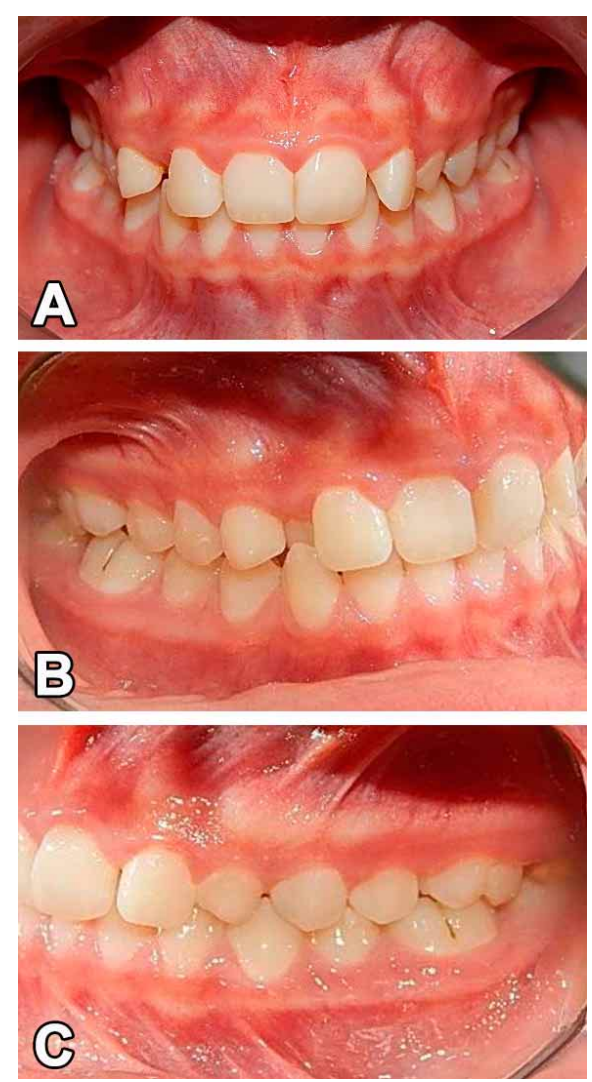

Fig. 2. A. Vista intraoral frontal. Fig. 2. B. Vista lateral derecha. Fig. 2. C. Vista lateral izquierda.

Fue necesario el control en este paciente por lo que se indicó la toma de las radiografías periapicales una vez al mes, con el fin de dar seguimiento y para controlar cualquier alteración, en Fig. $5 \mathrm{~A}$ se ven los brackets y la ligadura con el canino traccionado. En Fig. 5B, la fecha de revisión en el mes de noviembre y en Fig. 5C, la revisión de diciembre. 

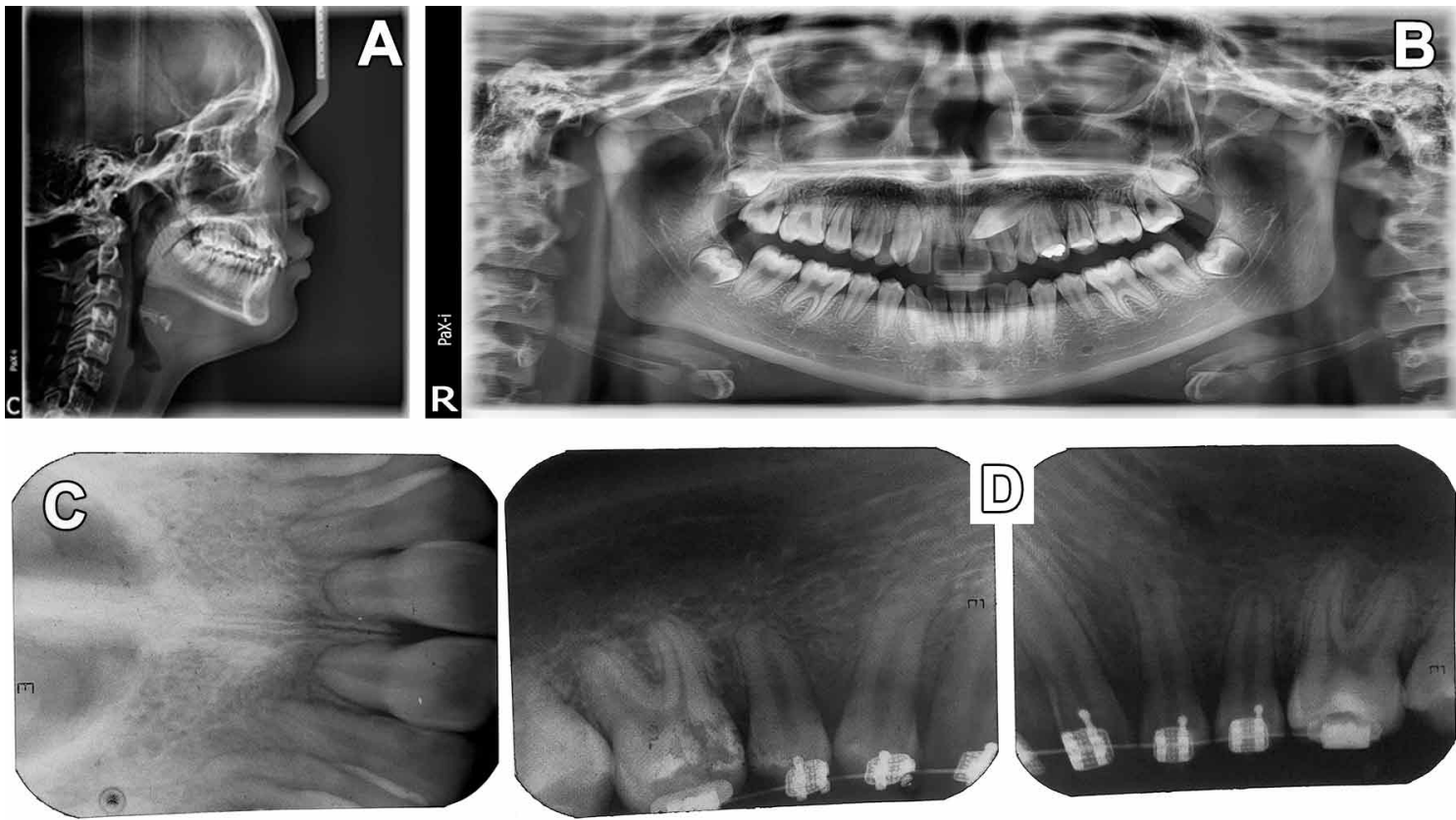

Fig. 3.A Radiografia lateral de craneo inicial. Fig. 3.B. Radiografia panoramica. Fig. 3.C Radiografía centrales superiores. 3.D Radiografía periapical de zona de premolares.
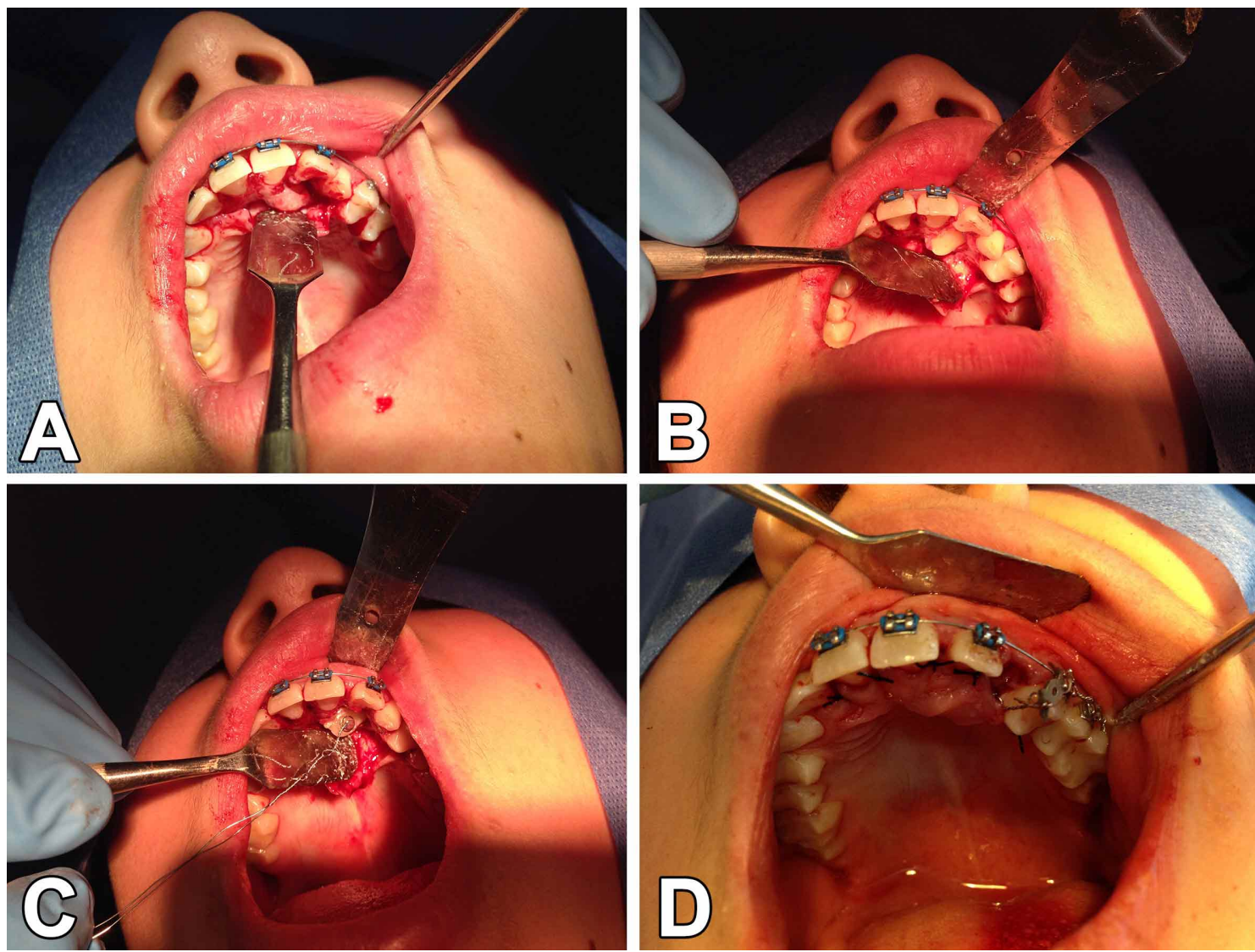

Fig. 4A. Desprendiendo paladar. Fig. 4B. Levantamiento de colgajo. Fig. 4C.Tracción quirúrgica del canino 23. Fig. 4D. Sutura del paladar. 

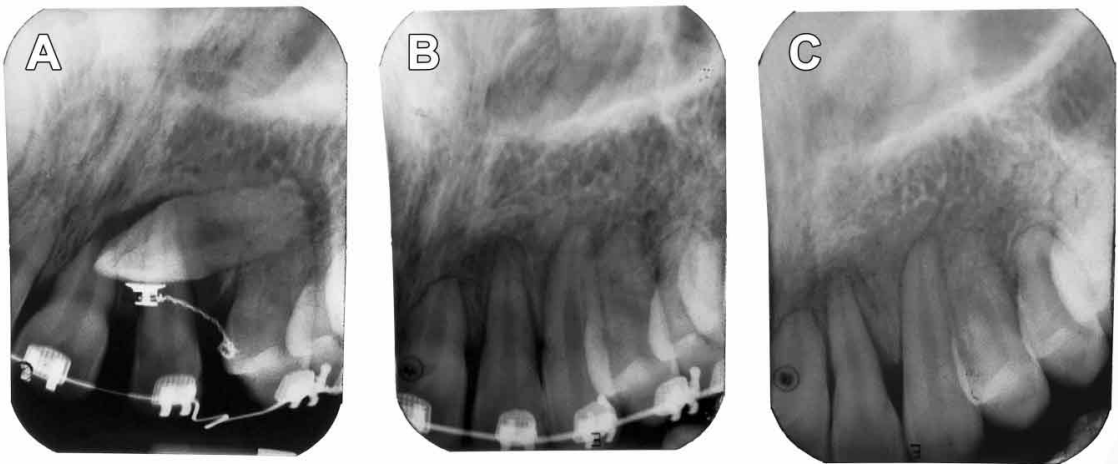

Fig. 5A. control radiográfico Octubre 22 oct. 5B. control radiográfico Noviembre. 5D. control radiográfico Diciembre.
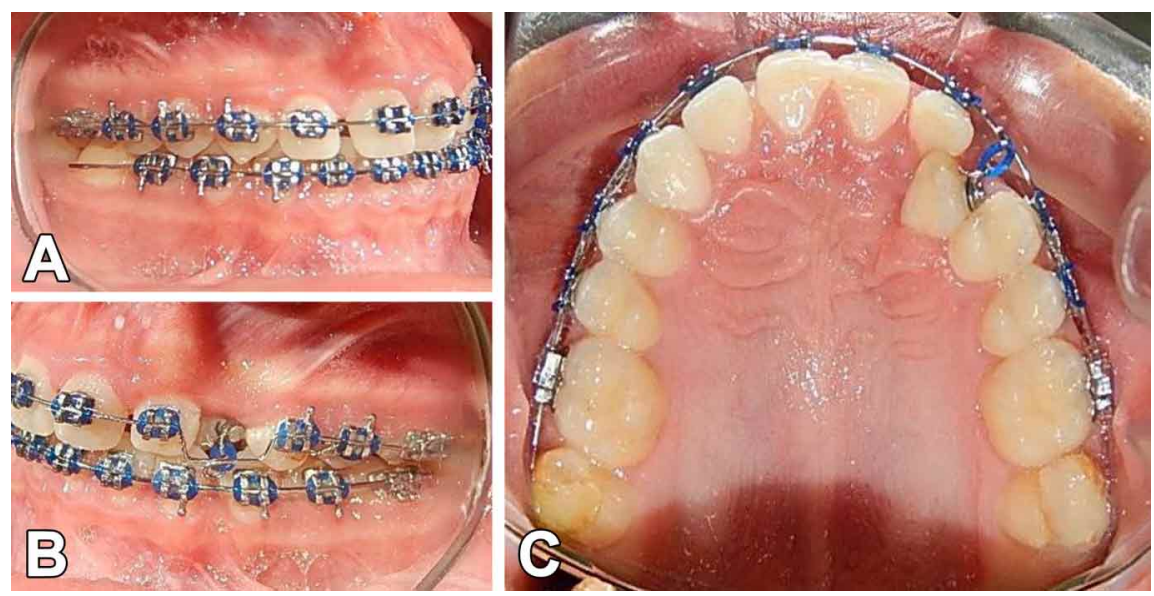

Fig. 6A. aparatos de ortodoncia instalados vista intraoral derecha. 6B. aparatos de ortodoncia instalados vista intraoral izquierda. 6C. aparatos de ortodoncia instalados vista oclusal superior del canino.
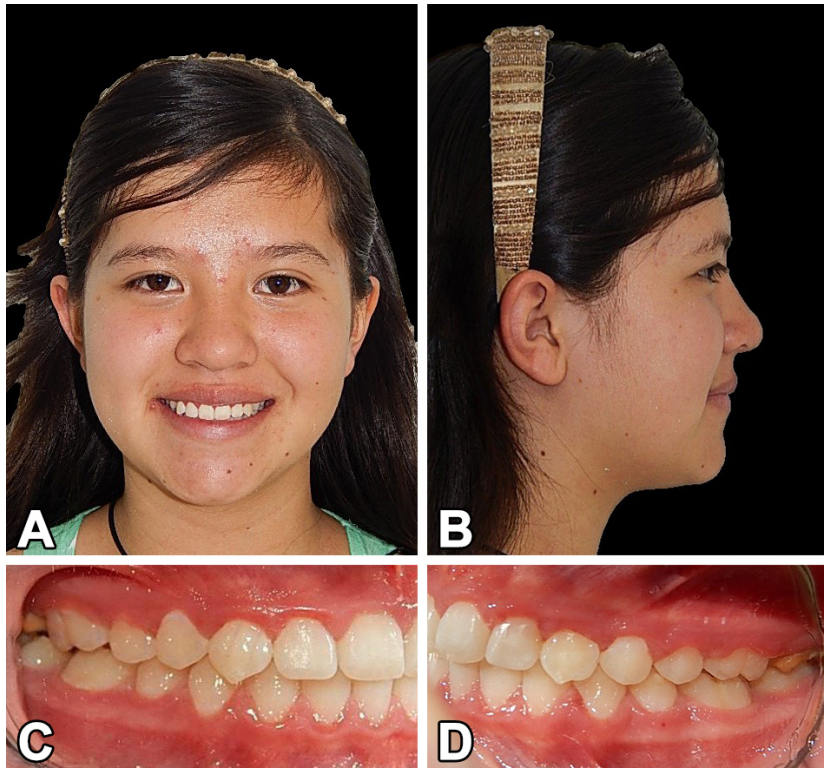

Fig. 7A. Vista extraoral frontal. 7B. Vista extraoral lateral. 7C. Vista intraoral lateral derecha. 7D. Vista intraoral lateral izquierda.
A la paciente se le indicó la extracción de los caninos temporales (53-63), una vez llevadas a cabo se continuó con la alineación nivelación, la tracción quirúrgica del 23, se cerraron espacios. Para el detallado, y retención. Se utilizaron los arcos de Nitinol 0,012, 0,014, 0,018 superior e inferior, arcos de acero 0,018 y 0,020 . Se colocaron los bite turbos en los dientes 36 y 46 ; se puso un botón bondeable, con ligadura metálica, y la cadena elastómera a nivel del d 23. Se realizaron dobleces de primer, segundo y tercer orden. Los estudios de progreso en las fotografías intraorales fueron tomados después de los 10 meses de haberse iniciado el tratamiento; en la fotografía intraoral lateral derecha se puede observar la incorporación al arco y la corrección de la mordida cruzada del OD 13 , y la relación de clase I canina en la lateral derecha (Fig. 6A) En la lateral izquierda se observa la incorporación al arco del diente 23 mediante un módulo elastómero (Fig. 6B) y en la oclusal superior, la presencia del 23 en la cavidad bucal todavía un poco cargado en paladar (Fig. 6C).

Estudios finales: El caso fue finalizado después de 1 año 2 meses de tratamiento. En el análisis extraoral, tenemos como resultado una paciente con una sonrisa agradable y armónica, como lo vemos en Fig. 7A; y un perfil facial recto (Fig. 7B). En las fotografías intraorales observamos el lugar correcto del canino alineado, derecha, clase I canina derecha y molar derecha y lado izquierdo (Fig. 7C) clase I canina izquierda y molar izquierda. Y una comparativa del inicio del tratamiento al final para ver la forma de los arcos (Fig. 7D.)

La paciente termina en clase I canina bilateral y clase I molar bilateral derecha, fue muy colaboradora durante su tratamiento, sigue en control. Para la retención se confeccionaron retenedores "Hawley" superior e inferior con "Finger" a nivel de los caninos 13 y 23 , (Figs. $8 \mathrm{~A}, 8 \mathrm{~B}, 8 \mathrm{C}$.) 

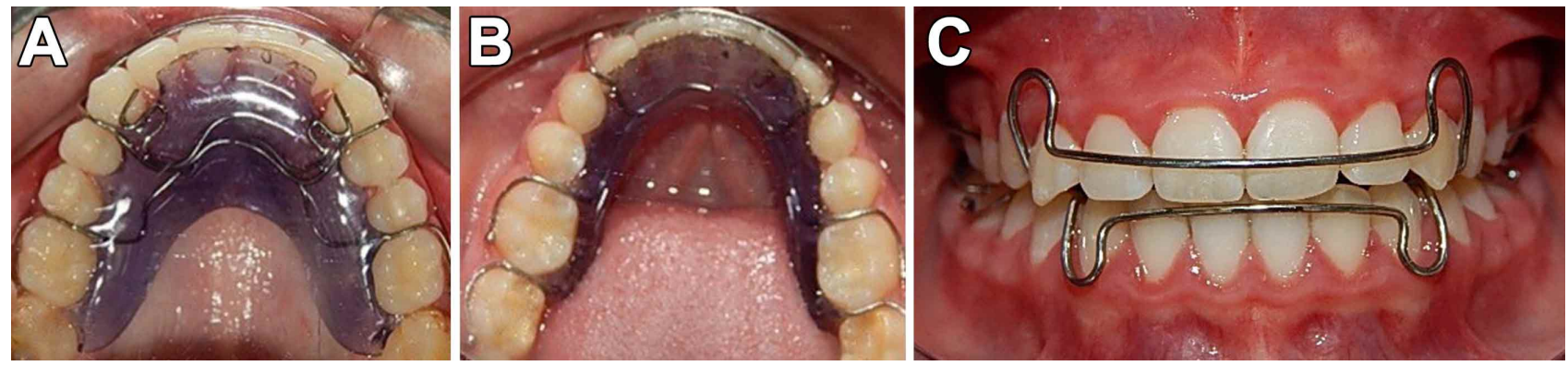

Fig. 8. A. Retenedor Hawley superior. 8 B. Retenedor Hawley inferior. 8 C. Retenedores vista frontal.

\section{DISCUSIÓN}

Proffit \& Ugalde refieren a la retención dental como "a un estado en el cual un diente, parcial o totalmente desarrollado, queda alojado en el interior de los maxilares después de haber pasado la época promedio normal de su erupción" (Rodriguez; Proffit \& Ugalde). A este respecto Uribe manifiesta que son más comunes los caninos retenidos maxilares que los mandibulares (Uribe). Como fue el caso del CESO presentado. Por otro lado coincidimos con Gómez \& Jaramillo, quienes señalan que para la tracción de caninos es necesario una evaluación cuidadosa del estado de desarrollo de la dentición (Gange). En cuanto al pronóstico de un diente retenido, este va a obedecer a una variedad de factores, tales como: la posición del diente retenido con respecto a los dientes vecinos, su angulación, la distancia que el diente debe recorrer y la posible presencia de anquilosis, como lo refieren Gange, Nozawa et al. (2003), Jacobs (2000), Ericson \& Kurol (2000), Chatem (2004) y Chapokas et al. (2011). En este caso se siguieron tres actitudes ante la detección de la retención, abstención, exodoncia o recolocación del diente retenido en la arcada, mediante procedimientos quirúrgicos-ortodóncico, las cuales a su vez dependieron de diversos factores como: edad del paciente, grado de desarrollo de su dentición, la posición del canino no erupcionado, la evidencia de reabsorción radicular de los incisivos permanentes, la percepción del problema por parte del paciente y la cooperación que el mismo pueda prestar durante el tratamiento.

\section{CONCLUSIÓN}

Después de haber realizado una revisión exhaustiva y análisis detallado de los aspectos más importantes de la retención de los caninos se concluye que, no es rara la presencia de caninos retenidos, presentándose con mayor incidencia en el maxilar superior, con ubicación palatina, tendencia unilateral del lado izquierdo y se observa principalmente en mujeres.

Es fundamental realizar una correcto y minucioso diagnóstico, por medio de exámenes clínicos, radiográficos y tomográficos, ya que nos permite detectar, cualquier alteración de la erupción a una edad temprana, aproximadamente a los ocho años. Además, un acertado diagnóstico nos permitirá elaborar un adecuado plan de tratamiento y de esta manera, resolver rápida y efectivamente cualquier problema, evitando complicaciones que comprometan la integridad del resto de los dientes, como lo es la resorción radicular. Errores en el diagnóstico e interpretación de los caninos con el potencial de retención, pueden llevar a serias malposiciones dentarias que posteriormente requieran de un tratamiento de ortodoncia prolongado.

OROZCO, E. E.; GURROLA M. B. \& CASASA, A. A. Traction of left maxillary impacted canine with bondeable button, metal ligature and elastomeric chain. Int. J. Odontostomat., 11(1):77-82, 2017.

ABSTRACT: Patient with 13 years and 7 months old, the reason for visit "a fang on the palate" presenting molar class I bilateral, right canine class I with OD 53, right class II canine with 13 , left canine Class I, is a class I skeletal, normodivergent, with the adequate overbite and overjet, superior moderate crowding and lower light, cross bite between teeth: canine 13 and 43, 23 impacted upper right canine, root shortening $11,21,35,45$. Treatment consisted of extraction of primary teeth 53-63, alignment, leveling, surgical traction 23, space closure, detailed, and retention. Retention by upper and lower Hawley retainers with fingers in canine 1323 . The active treatment time was 1 years 2 months.

\section{KEY WORDS: impacted canine, surgical traction.}


OROZCO, E. E.; GURROLA M. B. \& CASASA, A. A. Tracción de canino maxilar izquierdo impactado con botón bondeable, ligadura metálica y cadena elastomérica. Int. J. Odontostomat., 11(1):77-82, 2017.

\section{REFERENCIAS BIBLIOGRÁFICAS}

Ballesteros, D. Curso Cirugía Virtual. Bogotá, Universidad Nacional de Colombia Bogotá, Facultad de Odontología, 2016. Disponible en: http://www.virtual.unal.edu.co/cursos/odontologia/ 2005168/lecciones/Capitulo1/Lec1.html.Consulta julio 2016.

Chapokas, A. R.; Almas, K. \& Schincaglia, G. P. The impacted maxillary canine: a proposed classification for surgical exposure. Oral Surg, Oral Med. Oral Pathol, Oral Radiol. Endodon., 113(2):222-28, 2011.

Chatem, R. Maxillary canine impaction; a final twist in the tale? J. Orthod., 31(1):13-14, 2004

Ericson, S. \& Kurol, J. Resorption of incisors after ectopic eruption of maxillary canines: a CT study. Angle Orthod., 70(6):415-23, 2000.

Esquivel, J. L. Dientes Retenidos. Publicaciones Universidad de Costa Rica, 3-8, 1978.

Gange, R. J. Attachment of elastomeric thread to a palatally impacted cuspid. J. Clin. Orthod., 28(8):458, 1994.

Gómez, S. L. \& Jaramillo, P. A. Manejo ortodoncico de caninos maxilares retenidos. Rev. Fac. Odontol. Univ. Antioquia, 13(2):79-85, 2002.

Jacobs, S. G. Radiographic localization of unerupted teeth: further findings about the vertical tube shift method and other localization techniques. Am. J. Orthod. Dentofacial Orthop., 118(4):439-47, 2000.

Lucea, A. Caninos incluidos. Tratamiento con biomecánica de arcos dobles. Rev. Ortod. Clin., 8(1):22-32, 2005.

Nozawa, T.; Sugiyama, T.; Yamaguchi, S.; Ramos, T.; Komatsu, S.; Enomoto, H.; \& Ito, K. Buccal and coronal bone augmentation using forced eruption and buccal root torque: a case report. Int. J. Periodontics Restorative Dent., 23(6):585-91, 2003.

Proffit, W. R.; Fields, H. W. \& Sarver, D. M. Ortodoncia Contemporánea. $4^{\mathrm{a}}$ ed. Barcelona, Elsevier, 2008.

Ugalde, M. F. J. Clasificación de caninos retenidos y su aplicación clínica. Rev. A. D. M., 58(1):21-30, 2001.

Uribe-Restrepo, G. Ortodoncia, Teoría y Clínica. $2^{a}$ ed. Medellín, Corporación para Investigaciones Biológicas, 2009.
Dirección para correspondencia:

Dra. Beatriz Gurrola

Profesora del CESO

México

E-mail: beatgurrola@gmail.com

Recibido : 22-09-2016

Aceptado: 23-12-2016 